\title{
Research Square \\ A Continuum Deformation Approach for Growth Analysis of COVID-19 in the United States
}

Sadra Hemmati ( $\square$ shemmati@villanova.edu )

Villanova University https://orcid.org/0000-0003-0793-4677

Hossein Rastgoftar

Villanova University https://orcid.org/0000-0001-9212-7161

\section{Research Article}

Keywords: COVID-19, pandemic, growth analysis

Posted Date: March 16th, 2021

DOl: https://doi.org/10.21203/rs.3.rs-310937/v1

License: (9) This work is licensed under a Creative Commons Attribution 4.0 International License. Read Full License

Version of Record: A version of this preprint was published at Scientific Reports on September 8th, 2021. See the published version at https://doi.org/10.1038/s41598-021-97021-z. 


\title{
A Continuum Deformation Approach for Growth Analysis of COVID-19 in the United States
}

\author{
Sadra Hemmati ${ }^{1, *}$ and Hossein Rastgoftar ${ }^{1, *}$ \\ ${ }^{1}$ Villanova University, Mechanical Engineering Department, Pennsylvania 19085, United States \\ *shemmati@villanova.edu, hossein.rastgoftar@villanova.edu
}

\begin{abstract}
The COVID-19 global pandemic has significantly impacted every aspect of life all over the world. The United States is reported to have suffered more than $20 \%$ of the global casualties from this pandemic. It is imperative to investigate the growth dynamics of the disease in the US based on varying geographical and governmental factors that best manifest itself in each state of the country. This paper utilizes a hybrid machine learning and continuum deformation-based approach for analyzing the stability of the rapid COVID-19 growth. The proposed continuum deformation model is used to learn the parameters of pandemic growth based on the training data of total cases, deaths, and recoveries in each state of the United States from March 12, 2020 to January 28, 2021. Using this approach, multiple periods of the nationwide and State-level pandemic growth patterns are discovered and analyzed.
\end{abstract}

\section{Introduction}

The first death caused by COVID-19 in the United States is believed to have occurred in Santa Clara County, California on the February, 6th, and the virus has rapidly grown across the country since then. Studies have shown that the virus is dominantly transmitted through close contact with infected people and contaminated surfaces as well as respiratory droplets ${ }^{3,5}$. This spread includes a dispersion dynamics, and trajectory tracking techniques in the field of Control Theory can be effectively utilized.

Usually, the daily reports of pandemic statistics only include the deaths, total cases, active cases, and other similar explicit demographic parameters for varying geographical locations. Thus, models that can learn well from these types of data sets to infer the spread dynamics are very valuable and versatile for State-level decision making. Considering the different scales of analysis (global, between two countries, inside countries, between all States of a country, between Counties of a State, etc), obtaining detailed information regarding factors that affect transmission of the disease are challenging.

\subsection{Related Work}

Different types of models with varying theoretical principles have been used for pandemic growth prediction and stability analysis. The methods range form statistical inference and correlation, to differential equation-based models. SIR method ${ }^{4,6}$, SEIR dynamics ${ }^{7,8}$, Metapopulation Dynamics ${ }^{9,10}$, and Mean-Field Theory ${ }^{11,12}$ are examples of existing models used to estimate the dynamics of infectious diseases. $\mathrm{In}^{14}$, the authors applied the SIR model to estimate and evaluate the negative impacts of the growth of COVID 19 in the US. Metapopulation Dynamics is used in ${ }^{13}$ to evaluate the impact of domestic and international travel on the spread of COVID-19. The SEIR model was used to analyze the epidemic of COVID-19 in mainland China ${ }^{15}$ and the Diamond Princess cruise ship ${ }^{16}$. In ${ }^{12}$, the authors apply mean-field theory to analyze and predict the spread of COVID-19 in China, Italy, and France. Alternatively, in $^{5}$, the authors applied a Bayesian regression model to analyze the aerosol stability of COVID-19. $\mathrm{In}^{17}$, the authors discuss the estimation of the growth rate of a pandemic using maximum likelihood, using a data bank. Rastgoftar utilized a mass-conservation based integrated data into physics-based finite-time estimation model to analyze stability of the rapid COVID-19 growth in the United States ${ }^{2} . \mathrm{In}^{18}$, the authors, using the Moving Regression (MR) technique and a Hidden Markov Model (HMM), aimed at developing a simple framework for prediction of the growth rate (cases/day) and growth acceleration (cases/day ${ }^{2}$ ) of COVID-19 cases in real-time and study the effects of public health measures on the prevalence of COVID-19. Another correlation study aimed at studying the seasonal temperature variations on pandemic growth. The authors report their developed model can explain 36\% of the variation in maximum COVID-19 growth rates based on weather and demography $(17 \%)$ and country-specific effects $(19 \%)^{19}$. In ${ }^{20}$, the authors model the US epidemic at the State-level, using publicly available death data within a Bayesian hierarchical semi-mechanistic framework in which the SARS-COV2 transmission was predicted using mobility trends. It is reported that Statewide stay-at-home orders had the strongest causal impact on reducing social interaction and mobility. For instance, $\mathrm{in}^{21}$, the authors report that the Statewide stay-at-home orders result in a steady decline in confirmed cases, starting from ten days after implementation and reaching a $37 \%$ decrease after fifteen days, consistent with the testing practices and incubation period of the disease. They mention this 


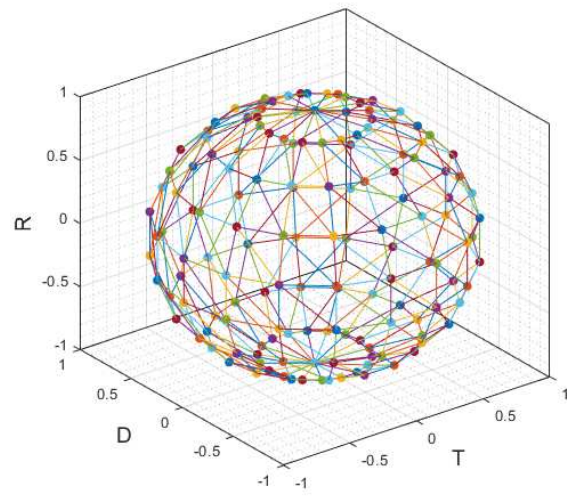

(a)

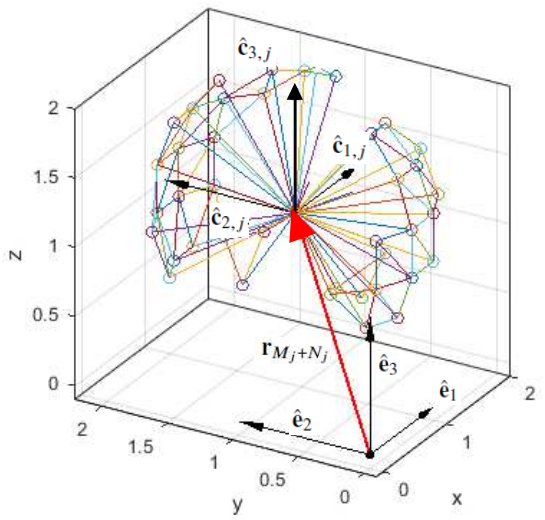

(b)

Figure 1. (a) $T-D-R$ space polyhedralization for $j \in C$, using the Eq. (9a) and Eq. (9b). The polytopes are contained in a sphere of radius 1 at the initial moment, and conserve their orientation and are deformed and elongated during the pandemic growth. (b) The containment polytope $1 \in C$ at day $k=100$. To determine the containment polytope we choose $p=15$ and $q=27$, therefore, $\mathcal{B}_{1}=\{1,2, \ldots, N-1\}$ and $\mathcal{I}_{1}=\{N\}$. The origin of the local coordinate of polytope $1 \in C$ is positioned at $\overline{\mathbf{r}}_{N, g}=1.1 \times 10^{4} \hat{\mathbf{e}}_{1}+1.3 \times 10^{4} \hat{\mathbf{e}}_{2}+1.2 \times 10^{4} \hat{\mathbf{e}}_{3}$, therefore $\mu_{T, 1}=1.1 \times 10^{4}, \mu_{D, 1}=1.3 \times 10^{4}$, and $\mu_{R, 1}=1.2 \times 10^{4}$ (See Remark $1)$.

executive order had the strongest causal impact on reducing social interactions. Therefore, we examine our results with the stay-at-home executive orders of each State.

\subsection{Contributions and Outline}

This paper integrates the principles of continuum mechanics with machine learning algorithms to effectively model and learn the growth of a pandemic disease. We treat evolution of a pandemic disease as a continuum deformation problem in three dimensional $T-D-R$ space where $T, D$, and $R$ denote the total number of infected cases, the total number of the deaths, and the total number of recoveries, respectively. We focus on the growth of COVID-19 in the US States and the District of Columbia. Hence, the pandemic continuum consists of 51 particles of 3-D deformable bodies evolving in the $T-D-R$ space.

The main objective of this paper is to analyze the growth of the pandemic disease by analyzing deformation of the pandemic continua in the $T-D-R$ space. To this end, we first apply the k-means algorithm, divide the US States/district into a finite number of clusters and determine the centroid of each cluster in the $T-D-R$ space. We then offer a novel polyhedral learning approach to contain each cluster by a 3-D polytope. We apply the principles of continuum mechanics to analyze the growth of COVID-19 pandemic by evaluating the deformation of the containment polytope.

Compared to the existing research and the authors' previous work, this paper offers the following novel contributions:

1. The existing learning methods solve a nonlinear optimization problem to determine the solution of a classification problem. Therefore, the solution of a classification problem may not necessarily converge to the global optimum. However, the proposed polyhedral learning does not deal with the convergence issue of the existing approaches since it determines the boundary of the containment polytopes by assigning maxima of finite sets of discrete variables.

2. The proposed polyhedral learning method ensures that the training data are all enclosed by the containment polytopes.

3. To the best of our knowledge, this is the first paper that models evolution of a pandemic disease as a continuum deformation coordination.

This paper is organized as follows: A polyhedralization method is developed in Section 2 and followed by the polyhedral learning in Section 3. Pandemic disease evolution is modeled as continuum deformation in Section 4. Results of stability analysis of pandemic evolution is discussed in Section 5. The conclusion is presented in Section 6.

\section{Motion Space Polyhedralization}

The pandemic grows in a 3-D space with coordinates $T, D$, and $R$ (previously defined) while the points in the space are clustered into $m$ groups. The evolving clusters are then contained by $m$ deformable polytopes in the $T-D-R$ space where 
identification numbers of the containment polytopes are defined by set $C=\{1,2, \cdots, m\}$. Configuration of every polytope $j \in C$ is determined by $N$ characteristic nodes and formed by $\rho$ tetrahedrons as described below.

\subsection{Characteristic Nodes of Polytope $j \in C$}

The geometry and location of polytope $j \in C$ is assigned by $N$ nodes in the $T-D-R$ space that are identified by set

$$
\mathcal{L}_{j}=\{(j-1) N+1, \cdots, j N\}
$$

Set $\mathcal{L}_{j}$ can be expressed as

$$
\mathcal{L}_{j}=\mathcal{B}_{j} \bigcup \mathcal{I}_{j}, \quad \forall j \in C
$$

where singleton $\mathcal{I}_{j}=\{j N\}$ defines the identification number of the interior characteristic node of polytope $j \in C$ and $\mathcal{B}_{j}=\mathcal{L}_{j} \backslash \mathcal{I}_{j}$ defines identification numbers of the boundary nodes of polytope $j \in C$.

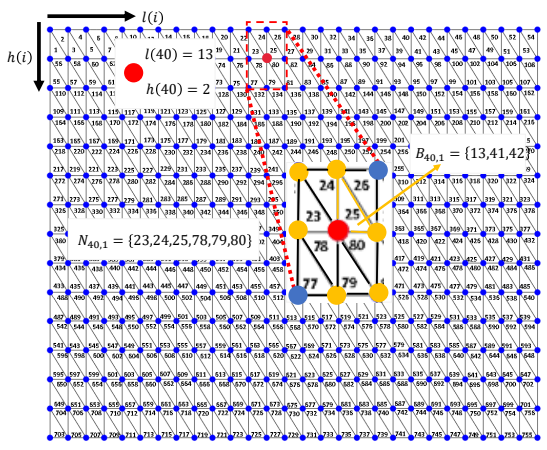

(a)

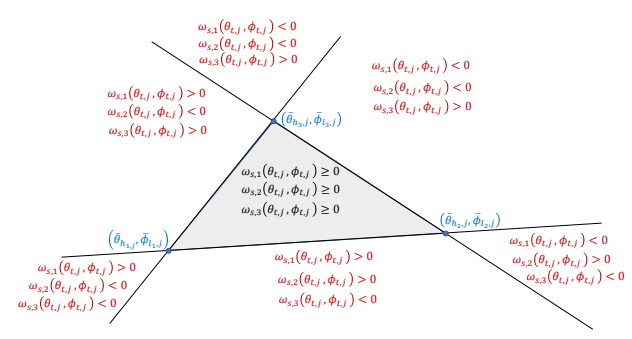

(b)

Figure 2. (a) The indexing method used to provide reference for each boundary node $i \in \mathcal{B}_{j}$ and $j \in C$ shown in Fig. 1(a). (b) Discretization of the $\theta-\phi$ plane based on the signs of components of vector $\boldsymbol{\Omega}_{s}\left(\theta_{t, j}, \phi_{t, j}\right)$ introduced in Eq. 20 .

\subsubsection{Local Coordinates of Characteristic Nodes}

Every index number $i \in \mathcal{B}_{j}$ can be converted to unique $l \in\{1, \cdots, p\}$ and $h \in\{1, \cdots, q\}$ coordinates for every $j \in C$. More specifically, $l: \mathcal{B}_{j} \rightarrow\{1, \cdots, p\}$ and $h: \mathcal{B}_{j} \rightarrow\{1, \cdots, q\}$ are defined as follows:

$$
\begin{aligned}
& \forall j \in C, i \in \mathcal{B}_{j}, \quad l(i)=\left\{\begin{array}{ll}
\left\lfloor\frac{i}{q}\right\rfloor+1 & \text { If } \left.i-q \mid \frac{i}{q}\right\rfloor \neq 0 \\
\left\lfloor\frac{i}{q}\right\rfloor & \text { If } \left.i-q \mid \frac{i}{q}\right\rfloor=0
\end{array},\right. \\
& j \in C, i \in \mathcal{B}_{j}, \quad h(i)=\left\{\begin{array}{ll}
i-q\left\lfloor\frac{i}{q}\right\rfloor & \text { If } i-q\left\lfloor\frac{i}{q}\right\rfloor \neq 0 \\
q & \text { If } i-q\left\lfloor\frac{i}{q}\right\rfloor=0
\end{array} .\right.
\end{aligned}
$$

On the other hand, $i \in \mathcal{B}_{j}$ can be defined based on positive integers $l \in\{1, \cdots, p\}$ and $h \in\{1, \cdots, q\}$ by

$$
i(l, h)=(l-1) q+h, \quad j \in C .
$$

\subsubsection{Local and Global Positions of Characteristic Nodes}

The global and local positions of node $i \in \mathcal{L}_{j}$ are denoted by $\overline{\mathbf{r}}_{i, g}$ and $\overline{\mathbf{r}}_{i, j, c}$, respectively. The global position is expressed with respect to the global coordinate system with fixed unit base vectors $\hat{\mathbf{e}}_{1}, \hat{\mathbf{e}}_{2}$, and $\hat{\mathbf{e}}_{3}$. Also, the local position of characteristic node $i \in \mathcal{L}_{j}$ is expressed with respect to local coordinate system of polytope $j$ whose base vectors are denoted by $\hat{\mathbf{c}}_{1, j}, \hat{\mathbf{c}}_{2, j}$, and $\hat{\mathbf{c}}_{3, j}$. Note that the origin of the local coordinate system of polytope $j \in C$ is located at $\overline{\mathbf{r}}_{j N, g}$, where $j N \in \mathcal{I}_{j}$ is the index number of the characteristic interior node of polytope $j \in C$. 
We assume that the local coordinate system of polytope $j \in C$ translates with no rotation in the $T-D-R$ space, thus $\hat{\mathbf{e}}_{1}=\hat{\mathbf{c}}_{1, j}=\left[\begin{array}{lll}1 & 0 & 0\end{array}\right]^{T}, \hat{\mathbf{e}}_{2}=\hat{\mathbf{c}}_{2, j}=\left[\begin{array}{lll}0 & 1 & 0\end{array}\right]^{T}, \hat{\mathbf{e}}_{3}=\hat{\mathbf{c}}_{3, j}=\left[\begin{array}{lll}0 & 0 & 1\end{array}\right]^{T}$ for every polytope $j \in C$. As a result, $\overline{\mathbf{r}}_{i, g}=$ $\bar{T}_{i, g} \hat{\mathbf{e}}_{1}+\bar{D}_{i, g} \hat{\mathbf{e}}_{2}+\bar{R}_{i, g} \hat{\mathbf{e}}_{3}$ and $\overline{\mathbf{r}}_{i, j, c}=\bar{T}_{i, j, c} \hat{\mathbf{c}}_{1, j}+\bar{D}_{i, j, c} \hat{\mathbf{c}}_{2, j}+\bar{R}_{i, j, c} \hat{\mathbf{c}}_{3, j}$ are related by

$$
\overline{\mathbf{r}}_{i, g}(k)=\overline{\mathbf{r}}_{j N, g}(k)+\overline{\mathbf{r}}_{i, j, c}(k), \quad \forall i \in \mathcal{B}_{j}, \forall j \in C,
$$

where $k$ is the number of days from the date of establishment of a pandemic disease (See Fig. 1(b)). Per Eq. (5), components of the global and local positions are related by

$$
\left\{\begin{array}{l}
\bar{T}_{i, g}(k)=\bar{T}_{j N, g}(k)+\bar{T}_{i, j, c}(k) \\
\bar{D}_{i, g}(k)=\bar{D}_{j N, g}(k)+\bar{D}_{i, j, c}(k) \\
\bar{R}_{i, g}(k)=\bar{R}_{j N, g}(k)+\bar{R}_{i, j, c}(k)
\end{array} \quad \forall i \in \mathcal{B}_{j}, \forall j \in C,\right.
$$

at day $k$. We express the local position of node $i \in \mathcal{B}_{j}$ by

$$
\overline{\mathbf{r}}_{i, j, c}=\bar{d}_{i, j} \hat{\mathbf{n}}_{i, j, c}
$$

where $\bar{d}_{i, j}$ is distance of boundary node $i \in \mathcal{B}_{j}$ from the characteristic interior node $j N \in \mathcal{I}_{j}$, and

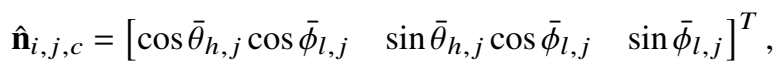

with latitude and azimuth angles

$$
\begin{aligned}
& \bar{\theta}_{h, j}=\frac{2 \pi h}{q}, \\
& \bar{\phi}_{l, j}=\frac{\pi(l-1)}{p-1},
\end{aligned}
$$

for $l \in\{1, \cdots, p\}$ and $h \in\{1, \cdots, q\}$. Thus, the direction unit vector $\hat{\mathbf{n}}_{i, j, c}$ is known for every boundary node $i \in \mathcal{B}_{j}$ and every cluster $j \in C$.

Remark 1. In the continuation of this paper, position of the characteristic interior node of polytope $j \in C$ is denoted

$$
\overline{\mathbf{r}}_{j N}=\mu_{T, j} \hat{\mathbf{e}}_{1}+\mu_{D, j} \hat{\mathbf{e}}_{2}+\mu_{R, j} \hat{\mathbf{e}}_{3}
$$

and assigned using the K-means clustering algorithm in Section 3.1, i.e. $\mu_{T, j}=T_{j N, j}, \mu_{D, j}=D_{j N, j}$, and $\mu_{R, j}=R_{j N, j}$ for every $j \in C$.

\subsection{Characteristic Tetrahedrons of Polytope $j \in C$}

The boundary of polytope $j \in C$ consists of $\rho=2 q(p-1)$ triangular cells, defined by set $\mathcal{S}_{j}=\{1, \cdots, 2 q(p-1)\}($ See Fig. 2(a)). For every $j \in \mathcal{C}$, set $\mathcal{S}_{j}$ can be expressed by

$$
\mathcal{S}_{j}=\mathcal{S}_{j, o} \bigcup \mathcal{S}_{j, e},
$$

where

$$
S_{j, o}=\{2 i(l, h)-1: l \in\{1, \cdots, p-1\}, h \in\{1, \cdots, q\}\} \quad \text { and } \quad S_{j, e}=\{2 i(l, h): l \in\{1, \cdots, p-1\}, h \in\{1, \cdots, q\}\},
$$

define triangular cells with odd and even identification numbers, respectively, where $i(l, h)$ is defined by Eq. (4) for given $l \in\{1, \cdots, p-1\}$ and $h \in\{1, \cdots, q\}$. Additionally, set $\mathcal{B}_{j}$ can be expressed by

$$
\mathcal{B}_{j}=\bigcup_{s \in \mathcal{S}_{j}} \mathcal{B}_{j, s}=\bigcup_{l=1}^{p-1} \bigcup_{h=1}^{q}\left(\mathcal{B}_{j, 2 i(l, h)-1} \bigcup \mathcal{B}_{j, 2 i}(l, h)\right), \quad \forall j \in C,
$$

where

$$
\mathcal{B}_{j, 2 i-1}= \begin{cases}\{i, i+q, i+q+1\} & \text { If } h(i)<q \\ \{i, i+q, i+1\} & \text { If } h(i)=q\end{cases}
$$




$$
\mathcal{B}_{j, 2 i}=\left\{\begin{array}{ll}
\{i, i+q+1, i+q+1\} & \text { If } h(i)<q \\
\{i, i+1, i-q+1\} & \text { If } h(i)=q
\end{array} .\right.
$$

Note that $\mathcal{B}_{j, s}$ defines the identification numbers of the vertices of triangle $s \in \mathcal{S}_{j}$ on the boundary of polytope $j \in C$. If $s \in \mathcal{S}_{j}$ is an odd number, Eq. (13a) defines vertices of triangular cell $s \in \mathcal{S}_{j}$. Otherwise, Eq. (13b) identifies vertices of triangle $s \in \mathcal{S}_{j}$.

For every node $i \in \mathcal{B}_{j}$ of polytope $j \in C$, set

$$
\mathcal{N}_{i, j}=\left\{s \in \mathcal{S}_{j}: i \in \mathcal{B}_{j, s}\right\},
$$

defines the index numbers of the triangular cells on the boundary of polytope $j \in C$ sharing common node $i \in \mathcal{B}_{j}$. Fig. 2(a) illustrates the configurations of the triangular cells on the boundary of every containment polytope $j \in C$ for $p=15$ and $q=27$. Fig. 2(a) also shows the nodes of triangular cell $25 \in \mathcal{S}_{j}$, defined by $\mathcal{B}_{j, 25}$, and the triangular cells sharing the common boundary node $40 \in \mathcal{B}_{j}$ and defined by set $\mathcal{N}_{40, j}$ for every $j \in C$, at day $k=100$. To determine the containment polytope, we choose $p=10$ and $q=18$. Therefore, $\mathcal{B}_{1}=\{1,2, \ldots, N-1\}$ and $\mathcal{I}_{1}=\{N\}$, where $N$ is 420 for this study. The origin of the local coordinate system of polytope $1 \in C$ is positioned at $\overline{\mathbf{r}}_{N, g}=1.1 \times 10^{4} \hat{\mathbf{e}}_{1}+1.3 \times 10^{4} \hat{\mathbf{e}}_{2}+1.2 \times 10^{4} \hat{\mathbf{e}}_{3}$, therefore $\mu_{T, 1}=1.1 \times 10^{4}$, $\mu_{D, 1}=1.3 \times 10^{4}$, and $\mu_{R, 1}=1.2 \times 10^{4}$ (See Remark 1$)$.

\section{Polyhedral Learning of a Pandemic Disease}

Let set $\mathcal{F}$ identifying a finite number of training data points be expressed by

$$
\mathcal{F}=\bigcup_{j \in C} \mathcal{F}_{j},
$$

where $\mathcal{F}_{1}$ through $\mathcal{F}_{m}$ are disjoint subsets of $\mathcal{F}$; set $\mathcal{F}_{j}=\left\{1, \cdots, f_{j}\right\}$ defines the training data points belonging to class $j \in C$. In this paper, set $\mathcal{F}$ defines a total of 51 triplet data points informing about the total number infected cases, deaths, and recoveries in 50 US States and the District of Colombia.

The data points provided by set $\mathcal{F}$ is used to determine the geometry of the containment polytopes deforming in the $T-D-R$ space. To this end, we first apply the K-means algorithm to cluster the US States and Washington DC into $m$ groups defined by $\mathcal{F}_{1}$ through $\mathcal{F}_{m}$ and to determine the centroids of clusters $\mathcal{F}_{1}$ through $\mathcal{F}_{m}$ denoted by $\overline{\mathbf{r}}_{j N} \in \mathcal{I}_{j} \subset \mathcal{F}_{j}$ for every $j \in C=\{1, \cdots, m\}$ (See Remark 1). Then, the data points belonging set $\mathcal{F}_{j} \backslash \mathcal{I}_{j}$ are used to determine the boundary of polytope $j \in C$ via assigning local positions $\overline{\mathbf{r}}_{(j-1) N+1}$ through $\overline{\mathbf{r}}_{j N-1}$.

\subsection{K-means Clustering Algorithm}

We utilize an unsupervised learning method to cluster the finite data points available by set $\mathcal{F}=\bigcup_{j \in C} \mathcal{F}_{j}$, into $m=|C|$ clusters, namely the K-means clustering, explained in Algorithm. 1. This algorithm works by calculating the distances of each data point to the center of cluster $j$. This method consists of two steps:

1. Cluster assignment step: In the first step of the Algorithm. 1, for each cluster $j \in C$, and $|C|=m$, a random vector (with the size of number of clusters, $m$ ) is generated to initialize the learning. Using the distance metrics appropriate to the data dimensions, the boundaries cluster $j$ sub-space is calculated.

2. Center update step: In the second step of the Algorithm. 1, the location of each centroid is updated using the newly generated boundaries from the step $i$ such that the cluster centers are moved to the average of each cluster points.

\subsection{Local Position Determination}

We denote the global and local positions of training data $t \in \mathcal{F}_{j}$ by $\mathbf{r}_{t, g}$ and $\mathbf{r}_{t, j, c}$ for every $j \in C$, where $\mathbf{r}_{t, g}=T_{t, g} \hat{\mathbf{e}}_{1}+D_{t, g} \hat{\mathbf{e}}_{2}+$ $R_{t, g} \hat{\mathbf{e}}_{3}$ and $\mathbf{r}_{t, j, c}=T_{t, j, c} \hat{\mathbf{c}}_{1, j}+D_{t, j, c} \hat{\mathbf{c}}_{2, j}+R_{t, j, c} \hat{\mathbf{c}}_{3, j}$ are related by

$$
\forall j \in C, \forall t \in \mathcal{F}_{j}, \quad \mathbf{r}_{t, j, c}=\mathbf{r}_{t, g}-\mathbf{r}_{j N, g} .
$$

The local position of training data point $t \in \mathcal{F}_{j}(j \in C)$ is expressed as follows:

$$
\mathbf{r}_{t, j, g}=d_{t, j, c}\left[\begin{array}{c}
\cos \theta_{t, j} \sin \phi_{t, j} \\
\sin \theta_{t, j} \sin \phi_{t, j} \\
\cos \phi_{t, j}
\end{array}\right],
$$




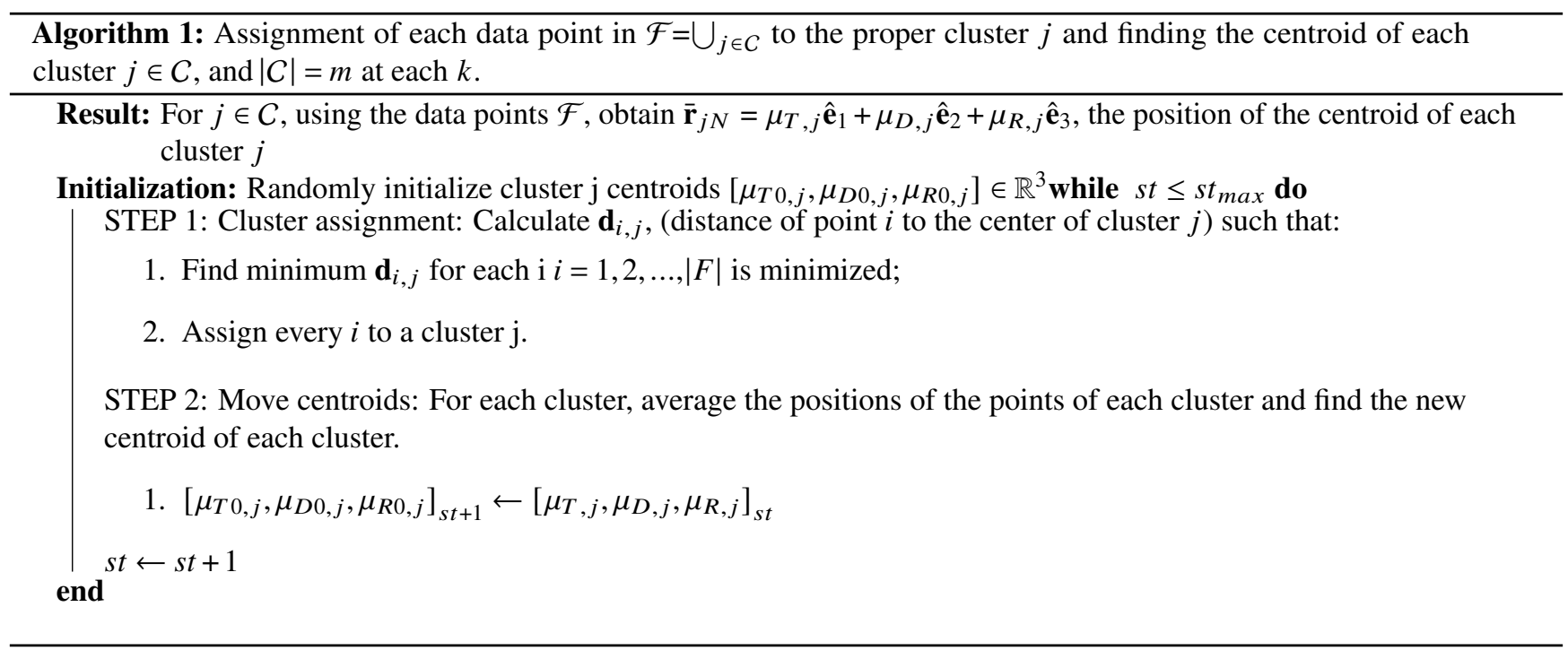

where

$$
d_{t, j}=\sqrt{T_{t, j, c}^{2}+D_{t, j, c}^{2}+R_{t, j, c}^{2}}, \quad \theta_{t, j}=\tan ^{-1}\left(\frac{D_{t, j, c}}{T_{t, j, c}}\right), \quad \phi_{t, j}=\cos ^{-1}\left(\frac{R_{t, j, c}}{\sqrt{T_{t, j, c}^{2}+D_{t, j, c}^{2}+R_{t, j, c}^{2}}}\right) .
$$

For every polytope $j \in C$, we determine the smallest polytope containing all training data points defined by set $\mathcal{F}_{j} \backslash \mathcal{I}_{j}$ by assiging the phase angle and radial distance of every training data as described below.

\subsubsection{Step 1: Phase Assignment}

Let $\beta_{j, s}=\left\{s_{1}, s_{2}, s_{3}\right\}$ define the vertices of triangular cell $s \in \mathcal{S}_{j}$ on the boundary of polytope $j \in C$. Given index numbers $s_{1} \in \mathcal{B}_{j}, s_{2} \in \mathcal{B}_{j}$, and $s_{3} \in \mathcal{B}_{j}$, we can use Eqs. (3a) and (3b) to obtain $l_{k}=l\left(s_{k}\right) \in\{1, \cdots, p\}$ and $h_{k}=h\left(s_{k}\right) \in\{1, \cdots, q\}$ for $k=1,2,3$. By invoking Eq. (7), we can write

$$
\overline{\mathbf{r}}_{s_{k}, j, c}=\bar{d}_{s_{k}, j} \overline{\mathbf{n}}_{s_{k}, j, c}
$$

where

$$
\overline{\mathbf{n}}_{s_{k}, j, c}=\left[\begin{array}{c}
\cos \theta_{h_{k}, j} \cos \phi_{l_{k}, j} \\
\sin \theta_{h_{k}, j} \cos \phi_{l_{k}, j} \\
\sin \phi_{l_{k}, j}
\end{array}\right]
$$

for $k=1,2,3$ where $s_{k} \in \mathcal{B}_{j, s} s \in \mathcal{S}_{j}$, and $j \in C$. We define vector function

$$
\boldsymbol{\Omega}_{s}\left(\theta_{t, j}, \phi_{t, j}\right)=\left[\begin{array}{c}
\omega_{s, 1}\left(\theta_{t, j}, \phi_{t, j}\right) \\
\omega_{s, 2}\left(\theta_{t, j}, \phi_{t, j}\right) \\
\omega_{s, 3}\left(\theta_{t, j}, \phi_{t, j}\right)
\end{array}\right]=\left[\begin{array}{ccc}
\bar{\theta}_{h_{1}, j} & \bar{\theta}_{h_{2}, j} & \bar{\theta}_{h_{3}, j} \\
\bar{\phi}_{l_{1}, j} & \bar{\phi}_{l_{2}, j} & \bar{\phi}_{l_{3}, j} \\
1 & 1 & 1
\end{array}\right]^{-1}\left[\begin{array}{c}
\theta_{t, j} \\
\phi_{t, j} \\
1
\end{array}\right]
$$

to determine the phase angle of the training data points define by set $\mathcal{F}$ according the following rules:

- If $\boldsymbol{\Omega}_{s}\left(\theta_{t, j}, \phi_{t, j}\right) \geq \mathbf{0}$, then, $\left(\theta_{t, j}, \phi_{t, j}\right)$ is inside the triangle with vertices $\left(\bar{\theta}_{h_{1}, j}, \bar{\phi}_{l_{1}, j}\right),\left(\bar{\theta}_{h_{2}, j}, \bar{\phi}_{l_{2}, j}\right)$, and $\left(\bar{\theta}_{h_{3}, j}, \bar{\phi}_{l_{3}, j}\right)$ (See Fig. 2(b)).

- If $\omega_{s, 1}\left(\theta_{t, j}, \phi_{t, j}\right), \omega_{s, 2}\left(\theta_{t, j}, \phi_{t, j}\right) \omega_{s, 3}\left(\theta_{t, j}, \phi_{t, j}\right)$ are not all non-negative, then, $\left(\theta_{t, j}, \phi_{t, j}\right)$ is outside the triangle with vertices $\left(\bar{\theta}_{h_{1}, j}, \bar{\phi}_{l_{1}, j}\right),\left(\bar{\theta}_{h_{2}, j}, \bar{\phi}_{l_{2}, j}\right)$, and $\left(\bar{\theta}_{h_{3}, j}, \bar{\phi}_{l_{3}, j}\right)$ 
Remark 2. By using vector function $\boldsymbol{\Omega}_{s}\left(\theta_{t, j}, \phi_{t, j}\right)$, we can express set

$$
\forall j \in C, \quad \mathcal{F}_{j}=\bigcup_{s \in \mathcal{S}_{j}} \hat{\mathcal{F}}_{j, s}
$$

where $\hat{\mathcal{F}}_{j, 1}, \cdots, \hat{\mathcal{F}}_{j, \rho}$ are disjoint subsets of set $\mathcal{F}_{j}$, and

$$
\hat{\mathcal{F}}_{j, s}=\left\{t \in \mathcal{F}_{j}: \boldsymbol{\Omega}_{s}\left(\theta_{t, j}, \phi_{t, j}\right) \geq \mathbf{0}\right\} \quad \forall j \in C, \forall s \in \mathcal{S}_{j}=\{1, \cdots, \rho\} .
$$

\subsubsection{Step 2: Assignment of Radial Distances of Boundary Nodes}

Let the training data $\operatorname{set} \mathcal{F}_{j}$ be expressed by

$$
\mathcal{F}_{j}=\bigcup_{i \in \mathcal{B}_{j}} \tilde{\mathcal{F}}_{j, i}=\bigcup_{i \in \mathcal{B}_{j}} \bigcup_{s \in \mathcal{N}_{i, j}} \hat{\mathcal{F}}_{j, s}, \quad j \in C
$$

where $\tilde{\mathcal{F}}_{j, i}$ defines all training data points that are enclosed by the tetrahedrons sharing the common node $i \in \mathcal{B}_{j}$ on the boundary of polytope $j \in C$. The polytope $j \in C$ encloses all training points, defined by set $\mathcal{F}_{j}$, if

$$
j \in C, \quad \bar{d}_{i, j}=\underset{t \in \tilde{\mathcal{F}}_{j, i}}{\operatorname{argmax}} \frac{1}{\mathbf{r}_{t, c, j} \cdot \hat{\mathbf{n}}_{i, j, c}}
$$

where $\hat{\mathbf{n}}_{i, j, c}$ is defined by (8) and “." is the dot product symbol.

\section{Pandemic Disease Evolution}

Evolution of polytope $j \in C$ in the $T-D-R$ space is defined by

$$
\mathbf{x}_{s, j}(k)=\mathbf{Q}_{s, j}(k) \mathbf{x}_{s, j, 0}+\mathbf{f}_{s, j}(k)
$$

for every tetrahedron $s \in \mathcal{S}_{j}$ at discrete time $k=1,2, \cdots$, where $k$ denotes the number of days from the establishment of a pandemic disease. For every $s \in \mathcal{S}_{j}$ and $j \in C$,

$$
\mathbf{Q}_{s, j}(k)=\left[\begin{array}{lll}
Q_{1,1, s, j}(k) & Q_{1,2, s, j}(k) & Q_{1,3, s, j}(k) \\
Q_{2,1, s, j}(k) & Q_{2,2, s, j}(k) & Q_{2,3, s, j}(k) \\
Q_{3,1, s, j}(k) & Q_{3,2, s, j}(k) & Q_{3,3, s, j}(k)
\end{array}\right] \in \mathbb{R}^{3 \times 3} \quad \text { and } \quad \mathbf{f}_{s, j}(k)=\left[\begin{array}{llll}
f_{1, s, j}(k) & f_{2, s, j}(k) & f_{3, s, j}(k)
\end{array}\right] \in \mathbb{R}^{3 \times 1}
$$

are non-singular Jacobian matrix and the rigid-body displacement vector, respectively. Note that $\mathbf{x}_{s, j, 0}=\left[\begin{array}{lll}T_{s, j, 0} & T_{s, j, 0} \quad R_{s, j, 0}\end{array}\right]^{T} \in$ $\mathbb{R}^{3 \times 1}$ is the reference position of interior point of tetrahedron $s \in \mathcal{S}_{j}$ of polytope $j \in C$ that is mapped to $\mathbf{x}_{s, j}(k)=\mathbf{x}_{s, j}(k)=$ $\left[T_{s, j}(k) \quad D_{s, j}(k) \quad R_{s, j}(k) \quad\right]^{T} \in \mathbb{R}^{3 \times 1}$ at day $k=1,2, \cdots$.

Assumption 1. In the reference configuration, boundary nodes of the polytope $j$ are all distributed on the surface of a unit sphere centered at the origin of the $T-D-R$ space. Reference configuration of every polytope $j \in C$ is shown in Fig. $1(a)$.

Remark 3. Although polytope $j \in C$ encloses all data points defined by set $\mathcal{F}_{j}, \mathbf{x}_{s, j, 0}=\left[\begin{array}{ccc}T_{s, j, 0} & T_{s, j, 0} \quad R_{s, j, 0}\end{array}\right]^{T} \in \mathbb{R}^{3 \times 1}$ and $\mathbf{x}_{s, j, 0}=\left[\begin{array}{lll}T_{s, j, 0} & T_{s, j, 0} & R_{s, j, 0}\end{array}\right]^{T} \in \mathbb{R}^{3 \times 1}$ do not necessarily assign positions of a data paint belonging to set $\mathcal{F}_{j}$. In other words, $\mathbf{x}_{s, j, 0}=\left[\begin{array}{lll}T_{s, j, 0} & T_{s, j, 0} & R_{s, j, 0}\end{array}\right]^{T} \in \mathbb{R}^{3 \times 1}$ and $\mathbf{x}_{s, j, 0}=\left[\begin{array}{lll}T_{s, j, 0} & T_{s, j, 0} & R_{s, j, 0}\end{array}\right]^{T} \in \mathbb{R}^{3 \times 1}$ can represent any arbitrary point inside the tetrahedron $s \in \mathcal{S}_{j}$ that is transformed under homogeneous transformation (25).

\subsection{Jacobian Matrix $\mathbf{Q}_{s, j}$ and Displacement Vector $\mathbf{f}_{s, j}$}

Let $\mathcal{B}_{s, j}=\left\{s_{1}, s_{2}, s_{3}\right\}$ and $\mathcal{I}_{j}=\{j N\}$ define index numbers of tetrahedron $s \in \mathcal{S}_{j}$ in polytope $j \in C$. For every $s \in \mathcal{S}_{j}$ and $j \in C$, positions of vertices of tetrahedron $s \in \mathcal{S}_{j}$ satisfy Eq. (25), thus we can write

$$
\begin{aligned}
& \overline{\mathbf{r}}_{s_{k}, g}(t)=\mathbf{Q}_{s, j}(k) \overline{\mathbf{r}}_{s_{k}, g, 0}+\mathbf{f}_{s, j}(k), \quad k=1,2,3, s_{k} \in \mathcal{B}_{s, j}, j \in C \\
& \overline{\mathbf{r}}_{j N, g}(t)=\mathbf{Q}_{s, j}(k) \overline{\mathbf{r}}_{j N, g, 0}+\mathbf{f}_{s, j}(k) .
\end{aligned}
$$


Per Assumption 1, $\overline{\mathbf{r}}_{j N, g, 0}=0_{3 \times 1}$ and

$$
\overline{\mathbf{r}}_{s_{h}, g, 0}=\hat{\mathbf{n}}_{s_{h}, j, c}, \quad h=1,2,3, s_{h} \in \mathcal{S}_{j}
$$

where the unit vector $\hat{\mathbf{n}}_{s_{h}, j, c}$ is defined by Eq. (8). Elements of matrix $\mathbf{Q}_{s, j}(k)$ and vector $\mathbf{f}_{s, j}(k)$ are then obtained as follows ${ }^{1}$ :

$$
\left[\begin{array}{c}
\operatorname{vec}\left(\mathbf{Q}_{s, j}(k)\right) \\
\mathbf{f}_{s, j}(k)
\end{array}\right]=\left[\begin{array}{ll}
\mathbf{I}_{3} \otimes \mathbf{L}_{0} & \mathbf{I}_{3} \otimes \mathbf{1}_{3 \times 1}
\end{array}\right]^{-1} \mathbf{p}(k)
$$

where "vec" is the matrix vectorization symbol,

$$
\begin{aligned}
& \operatorname{vec}\left(\mathbf{Q}_{s, j}(k)\right)=\left[\begin{array}{llll}
Q_{1,1, s, j} & \cdots & Q_{1,3, s, j} \cdots & Q_{3,3, s, j}
\end{array}\right]^{T} \in \mathbb{R}^{9 \times 1} \\
& \mathbf{p}(k)=\operatorname{vec}\left(\left[\begin{array}{llll}
{\left[\overline{\mathbf{r}}_{s_{1}, g}(k)\right.} & \overline{\mathbf{r}}_{s_{2}, g}(k) & \overline{\mathbf{r}}_{s_{3}, g}(k) & \overline{\mathbf{r}}_{j N, g}(k)
\end{array}\right]^{T}\right) \in \mathbb{R}^{12 \times 1} \\
& \mathbf{L}_{0}=\left[\begin{array}{llll}
\hat{\mathbf{n}}_{s_{1}, j, c} & \hat{\mathbf{n}}_{s_{2}, j, c} & \hat{\mathbf{n}}_{s_{3}, j, c} & \mathbf{0}_{3 \times 1}
\end{array}\right]^{T} \in \mathbb{R}^{4 \times 3} .
\end{aligned}
$$

\subsection{Eigen-decomposition of Pandemic Evolution}

We can use polar decomposition to express Jacobian matrix $\mathbf{Q}_{s, j}(k)$ by

$$
\mathbf{Q}_{s, j}(k)=\mathbf{R}_{s, j}(k) \mathbf{U}_{s, j}(k), \quad j \in C, s \in \mathcal{S}_{j},
$$

where $\mathbf{R}_{s, j}(k)$ is an orthogonal (rotation) matrix and $\mathbf{U}_{s, j}(k)$ is positive semi-definite at every discrete time $k$. Because every polytope $j \in C$ transforms without rotation, $\hat{\mathbf{e}}_{1}=\hat{\mathbf{c}}_{1, j}, \hat{\mathbf{e}}_{2}=\hat{\mathbf{c}}_{2, j}$, and $\hat{\mathbf{e}}_{3}=\hat{\mathbf{c}}_{3, j}$ for every cluster $j \in C, \mathbf{R}(k)=\mathbf{I}_{3}$ at every day $k$, and positive semi-definite matrix $\mathbf{Q}_{s, j}(k)=\mathbf{U}_{s, j}(k)$ assigns the linear deformation of tetrahedron $s \in \mathcal{S}_{j}$ in polytope $j \in C$.

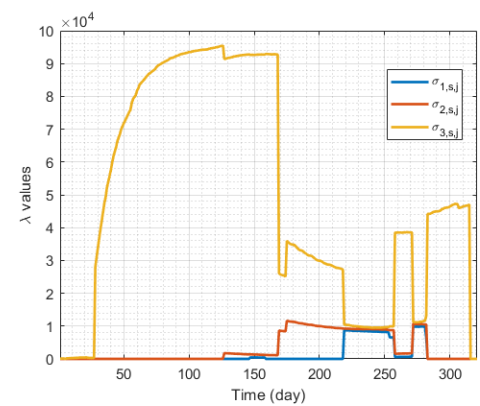

(a)

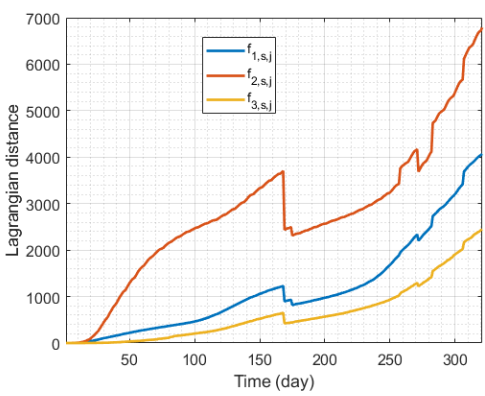

(b)

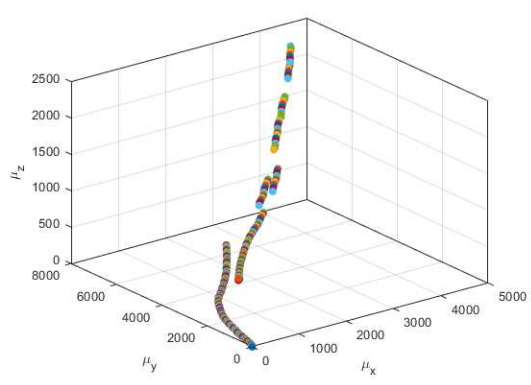

(c)

Figure 3. A two-cluster setting, $j \in C$ and $m=|C|=2$ (a) Eigenvalues $\lambda_{1}, \lambda_{2}$, and $\lambda_{3}$ of matrix $Q_{s, j}$ calculated for node 210 . For clustering, the Algorithm. 1 is used. (b) Distances of node 210 calculated for cluster $j=$ "2" (Eq. 24), where . (c) Position of the center of cluster "1" in the T-D-R space using the Algorithm. 1. Note that $\mathrm{j}=1 . \mu_{x}, \mu_{y}$, and $\mu_{z}$ stand for $\mu_{x, M_{j}+N_{j}}, \mu_{y, M_{j}+N_{j}}$, and $\mu_{z, M_{j}+N_{j}}$, respectively.

The eigenvalues of matrix $\mathbf{Q}_{s, j}$ are called the principal values of $\mathbf{Q}_{s, j}$, and are denoted by $\sigma_{1, s, j}, \sigma_{2, s, j}$, and $\sigma_{3, s, j}$. In constructing the Mohr's circle, $\sigma_{1, s, j}, \sigma_{2, s, j}$, and $\sigma_{3, s, j}$ are sorted such that:

$$
0 \leq \sigma_{3, s, j} \leq \sigma_{2, s, j} \leq \sigma_{1, s, j}
$$

Because matrix $\mathbf{Q}_{s, j}$ is only time varying, the principal values $\sigma_{3, s, j}, \sigma_{2, s, j}$, and $\sigma_{1, s, j}$ are spatially-invariant at every point of tetrahedron $s \in \mathcal{S}_{j}$. Given principal values of tetrahedron $s ß \mathcal{S}_{j}$, we define the following shear stress terms to analyze deformation the pandemic continuum:

$$
\tau_{1, s, j}(k)=\left(\sigma_{1, s, j}-\sigma_{3, s, j}\right) / 2, \quad \tau_{2, s, j}(k)=\left(\sigma_{1, s, j}-\sigma_{2, s, j}\right) / 2, \quad \text { and } \quad \tau_{3, s, j}(k)=\left(\sigma_{2, s, j}-\sigma_{3, s, j}\right) / 2 .
$$

Remark 4. Boundary nodes of tetrahedron $s \in \mathcal{S}_{j}$ are called active nodes and tetrahedron $s \in \mathcal{S}_{j}$ is called an active tetrahedron, if the volume of tetrahedron $s \in \mathcal{S}_{j}$ is nonzero. Therefore, $\sigma_{3, s, j}>0$, if $\mathcal{B}_{s, j}$ defines three active nodes on the boundary of polytope $j \in C$. 


\section{Stability Analysis}

In this section, we establish stability criteria and evaluate the stability of the pandemic growth using the principles of continuum mechanics. Intuitively, for any pandemic to be considered "stable", second time derivative of $T$ should be negative (meaning that the total cases are increasing but with a negative acceleration). However, this is not a sufficient condition. Additionally, the first time derivatives of $R$ should be greater than first time derivatives of $D$ and $T$, meaning that the "recovery speed" is greater than the death and infection rates. These dynamics criteria are captured using the continuum deformation-based Mohr's circle approach. Our stability analysis is complemented by defining different phases for the growth of COVID-192. "Phase 1" corresponds to the rapid spread of the virus in which the public health response relies on dramatic mitigation measures, like stay at home orders and social distancing, to slow the spread of the virus. "Phase 2" corresponds to flattening of the spread and the rise in the rate of infection is beginning to slow and stabilize. Hospitalizations and ICU bed usage continue to increase but are flattening "Phase 2". In "Phase 3" or recovery phase, the rate of infection is stable or declining. In "Phase 4" or revitalization, there is a continued decline in the rate of infection in new COVID-19 cases, and "Phase 5" corresponds to some kind of new normal situation and one could say the US is "restored" as far as COVID-19 pandemic is concerned.

We consider the spread of COVID-19 in the United States over 321 days from March 12, 2020 to January 28, 2021 where the number of infected cases (T), deaths (D), and recoveries (R) are obtained from ${ }^{23}$. We apply our proposed learning algorithm to analyze the $T-D-R$ time series data associated with every US State and District of Colombia and at each day of the pandemic. In addition, Statewide executive orders (shelter in place, travel restrictions, mask mandate, etc) are obtained ${ }^{24}$ for interpreting the results of our study. This US government website has provided a manually curated dataset that provides a standardized view into State and county policy orders (executive orders, ordinances, etc.), the policy orders' effective dates, types, time of start and stop, etc. However, it mentions that "This data set is provided as-is. Due to the evolving nature of policy orders, the US Government makes no representations to its correctness or completeness." This point should considered when assessing the data presented $\mathrm{in}^{24}$.

Based on the mathematical foundation discussed in Section 4.2, eigen-decomposition of the pandemic evolution is performed and principal values of the active tetrahedrons are obtained and plotted in Fig. 5(a), Fig. 5(b), and Fig. 6(a). Visually, we can observe distinct regions of the

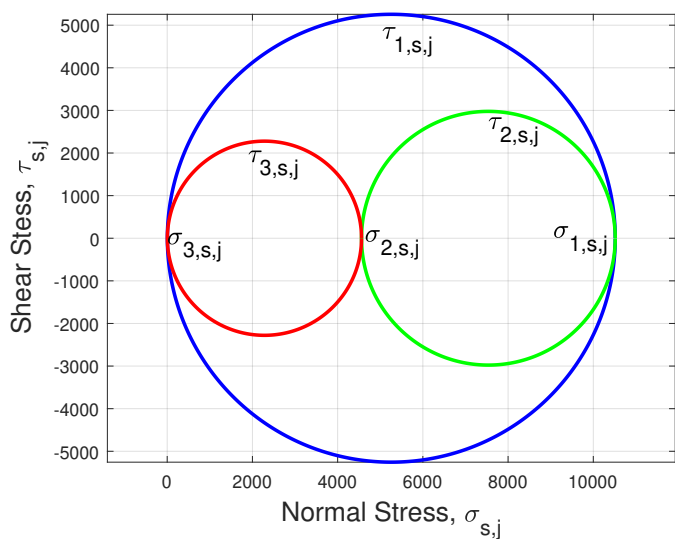

Figure 4. Sample Mohr's circle for node $S_{j}=80$ and for day $k=220$ and cluster $\mathrm{j}=1$ for $j \in C$ and $m=2$. pandemic growth, denoted by A, B, C, D, E, and F, respectively, based on time-series of maximal principal values of Jacobian matrix $\mathbf{Q}_{s, j}$ for every $s \in \mathcal{S}_{j}$ and $j \in C$. We use the term "region" because the word "phase" has distinct definition (as mentioned in Section 5 and has a different meaning in the context of the COVID-19 pandemic.

In general, there are five different growth stages of a pandemic: lagging (beginning of the outbreak), exponential (rapid growth), deceleration (growth decay), stationary (near zero growth), and linear growth (constant growth above zero) ${ }^{18}$. Looking at Fig. 5(b), we can identify region A to include the "lagging" (from day 0 to 20) and "exponential growth" (day 20 to 60). The region B can be associated with "growth decay", while region F can be associated with "linear growth". Regions C and D exhibit "stationary growth". The only region that exhibits a negative slope trend is region E.

In the following, the results related to two-cluster setting $(m=|C|=2)$, the principal value analysis, dynamics change, different regions of the pandemic based on continuum deformation (eigen-decomposition) are presented and discussed. For all cases, the discretization of the $T-D-R$ space is performed using $N=756$ tetrahedral mesh, created by $p=15$ and $q=27$ horizontal and vertical discretized points, in which the polytopes are allowed to elongate and deform without rotation.

As can be seen in Fig. 5(b), there is a dynamic shift around day 170 of the pandemic. This is the date that the centers of clusters experienced a "jump", as can be seen from Fig. 3(a) and Fig. 3(b). This can be attributed to (or correlated with) the Statewide executive orders that are important factors of changing the dynamics of the pandemic growth.

We hypothesize that clustering can group States by the extent to which they share similar COVID-19 growth dynamics. Based on two-cluster results, shown in Fig. 3(c), Fig. 6(b), and Fig. 6(a) we can see that the cluster 2 is a group of States with relatively higher adversary effect form the pandemic (or poorer performance for coping with the pandemic). We hypothesise that the two-cluster setting will group States in two-clusters: $i$ ) Critical, ii) Non-critical. The trend observed in Fig. 6(b) is that States of California (5), Florida (10), Illinois (14), Massachusetts (22), New Jersey (31), New York (33), Texas (44) and Washington (48) have been separated from the majority of States during the 321 days, and therefore they are considered to be in "critical" situation. One could define the two clusters as critical and noncritical. 


\section{Conclusion and Discussion}

In this work, we have adopted a new hybrid learning and continuum deformation framework to analyse the COVID-19 pandemic growth in the $T-D-R$ space. The $T-D-R$ space is discretized to create a finite set of nodes and tetrahedrons in which the characteristic polytopes of the training data can evolve in. if the volume of tetrahedron $s \in \mathcal{S}_{j}$ is nonzero, it means that it contains a data point. The maximal principal values of Jacobian matrix $\mathbf{Q}_{s, j}$ for every $s \in \mathcal{S}_{j}$ and $j \in C$ is found using the eigen-decomposition technique.

The magnitude of the principal stress values and their qualitative behaviour are the criteria we use for stability analysis. These magnitude of the values of principal stresses are quantitative values that are significantly correlated with the qualitative stability criteria (as defined by "Phases" of the pandemic by each State).

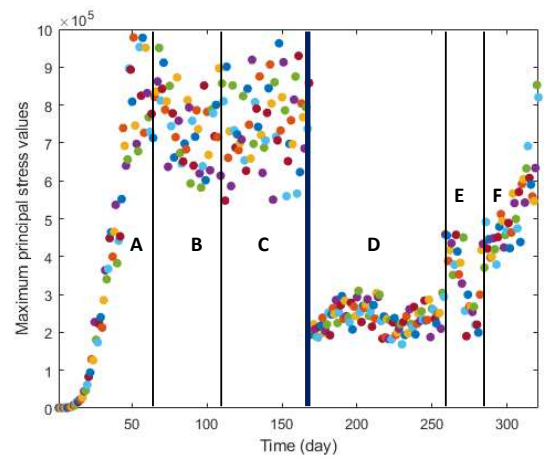

(a)

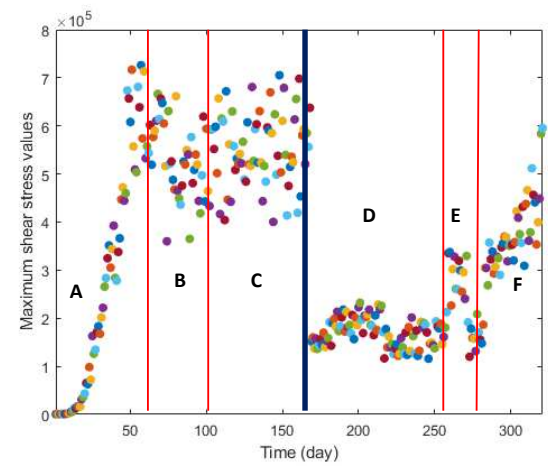

(b)

Figure 5. Daily time-series related to cluster $\mathrm{j} j \in C$ of (a) maximum values of principal stresses of $\sigma_{1, s, j}, \sigma_{2, s, j}$, and $\sigma_{3, s, j}$ for every active node, (b) maximum values of shear stresses of $\tau_{1, s, j}, \tau_{2, s, j}$, and $\tau_{3, s, j}$ for every node. Six regions of the pandemic in maximum shear stress plane based on the continuum deformation approach.

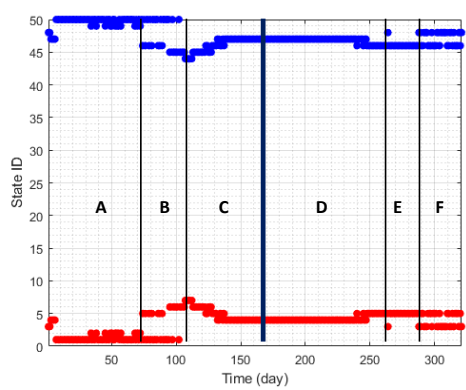

(a)

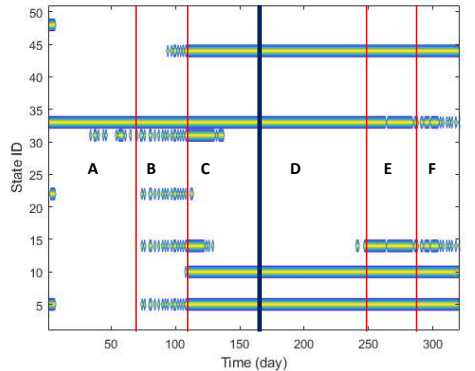

(b)

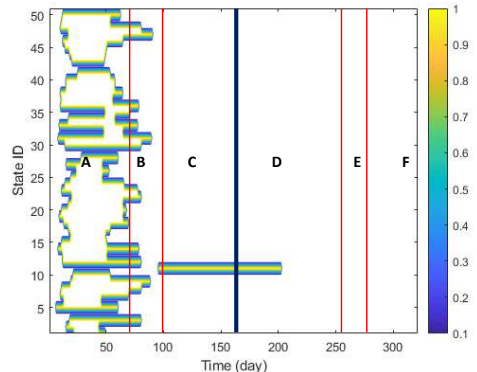

(c)

Figure 6. For a two-cluster setting ( $j \in C$, and $m=|C|=2$ ) (a) Maximum (blue) and minimum (red) values of numbers of States in clusters, (b) Time-series of cluster grouping for data $\mathcal{F}=\bigcup_{j \in C} \mathcal{F}_{j, k}$ in cluster for each time, $k$, (c) Time-series of "shelter in place" Statewide executive orders based on State ID $^{24}$.

The $T-D-R$ space of pandemic can be strongly specified by the principles of the continuum mechanics. Visually, in Fig. 6(b), we can observe six distinct regions of the pandemic growth based on time-series of cluster evolution from the K-means clustering algorithm, denoted by A, B, C, and D, E, and F, respectively. Our proposed continuum deformation and learning approach can capture different regions of the pandemic growth as can be seen from Fig. 6(b).

In the following, we discuss how well the stability criteria proposed in this work (Mohr's circle) in analysis of the pandemic growth matches with experimental real data of "shelter at place" executive order. Previous studies report that the most effective Statewide order to minimize virus spread is shelter at place ${ }^{21}$.

The initial exponential growth rate of an epidemic significantly determines its severeness ${ }^{17}$ and based on our stability criteria, this exponential growth happens in region A. The times series of shelter at place Statewide orders can be seen in Fig. 6(c) and for all States, except for Georgia, the shelter at place order happened at region A, and ended in region B. We 
can see there is an almost uniform reaction by the US States at the initial exponential phase of the pandemic, which is "rapid growth", therefore, this executive order has significantly helped the flattening of the virus spread as can be seen from Fig. 5(b).

The average duration of shelter in place order is 44.73 days with a standard deviation of 22.30 days. The States that has the highest duration of shelter at place were: Georgia 107 days, New Jersey 80 days, Virginia 73 days, and New York 68 days.

Our study has some limitation, for instance it is well-known that number of testing has direct impact on the $T-D-R$ numbers. In the US, only after 11 May 2020 (day 90 of our data set) was that the number of tests reached a reasonably high enough number (more than 375,000 tests are done each day). We found times of dynamics shift of COVID19 pandemic based on maximal values of the principal stresses of all nodes as a function of discrete time, i.e., every day of the pandemic that the data was available. Future research can look into the dynamics of pandemic growth of each region of the pandemic as determined by this work, in a more refined scale and using updated data, to uncover the causalities and correlations.

\section{Acknowledgement}

This work has been supported by the National Science Foundation under Award Nos. 1914581 and 1739525.

\section{Author contributions statement}

- SH carried out the simulation, developed and performed the continuum deformation analysis, and wrote Sections 1, 5, and 6.

- HR developed the polyhedal learning algorithm, provided the code for polyhedral learning, wrote Sections 2 and 3, and advised the work.

- The authors equally contributed in Section 4.

\section{Additional information}

The authors declare no competing interests.

\section{State IDs}

The IDs associated with each US State used for clustering is presented in Table. 1. The States are ordered alphabetically.

\begin{tabular}{|c|c|c|c|c|c|c|c|}
\hline State ID & State & State ID & State & State ID & State & State ID & State \\
\hline 1 & Alabama & 14 & Illinois & 27 & Nebraska & 40 & South Carolina \\
\hline 2 & Alaska & 15 & Indiana & 28 & Nevada & 41 & South Dakota \\
\hline 3 & Arizona & 16 & Iowa & 29 & New Hampshire & 42 & Tennessee \\
\hline 4 & Arkansas & 17 & Kansas & 30 & New Jersey & 43 & Texas \\
\hline 5 & California & 18 & Kentucky & 31 & New Mexico & 44 & Utah \\
\hline 6 & Colorado & 19 & Louisiana & 32 & New York & 45 & Vermont \\
\hline 7 & Connecticut & 20 & Maine & 33 & North Carolina & 46 & Virginia \\
\hline 8 & Delaware & 21 & Maryland & 34 & North Dakota & 47 & Wisconsin \\
\hline 9 & $\mathrm{DC}$ & 22 & Michigan & 35 & Ohio & 48 & Washington \\
\hline 10 & Florida & 23 & Minnesota & 36 & Oklahoma & 49 & West Virginia \\
\hline 11 & Georgia & 24 & Mississippi & 37 & Oregon & 50 & Wisconsin \\
\hline 12 & Hawaii & 25 & Missouri & 38 & Pennsylvania & 51 & Wyoming \\
\hline 13 & Idaho & 26 & Montana & 39 & Rhode Island & & \\
\hline
\end{tabular}

Table 1. The IDs associated with each US State used in this paper.

\section{References}

1. Rastgoftar, H., "Continuum Deformation of Multi-Agent Systems," Springer, 2016.

2. Rastgoftar, H., Atkins,E., "A Mass-Conservation Model for Stability Analysis and Finite-Time Estimation of Spread of COVID-19," IEEE Transactions on Computational Social Systems, article 9344853, pages=1-8, 2021, doi=10.1109/TCSS.2021.3050476. 
3. Centers for Disease Control and Prevention, "Coronavirus (COVID-19)," "https: / /www . cdc .gov/coronavirus / 2019-ncov/index.html", 2020.

4. Maier,B., and Brockmann, D. "Effective containment explains sub-exponential growth in confirmed cases of recent COVID-19 outbreak in Mainland China," arXiv preprint arXiv:2002.07572, 2020.

5. Van Doremalen, N. Bushmaker, T., Morris, D., Helbrook, M., Gamble, A., Williamson, B., Tamin, A., et. al, "Aerosol and surface stability of SARS-CoV-2 as compared with SARS-CoV-1," New England Journal of Medicine, Mass Medical Soc, 2020.

6. Kathakali, B., Parongama, S., "Space-time dependence of corona virus (COVID-19) outbreak", rXiv preprint arXiv:2003.03149, 2020.

7. Dukic, V., Lopes, H., Polson, NN, "Tracking epidemics with Google flu trends data and a state-space SEIR model," Journal of the American Statistical Association, volume 107, number 500, pages 1410-1426, 2012.

8. Liu, Y., Gayle, A., Wilder-Smith, A., and Rockl, J., "The reproductive number of COVID-19 is higher compared to SARS coronavirus," Journal of travel medicine, 2020.

9. Hanski, I., "etapopulation dynamics," Nature, volume 396, number 6706, pages 41-49, Nature Publishing Group, 1998.

10. Keeling, M, Bjrnstad, O.N., and Grenfell, B.T, "Metapopulation dynamics of infectious diseases," cology, genetics and evolution of metapopulations, Elsevier, pages 415-445, 2004.

11. Wu, Q., and Chen, S, "Mean field theory of epidemic spreading with effective contacts on networks," journal of Chaos, Solitons \& Fractals, pages 359-364, 2015.

12. Fanelli, D., and Piazza, F, "Analysis and forecast of COVID-19 spreading in China, Italy and France," journal of Chaos, Solitons \& Fractals, volume 134, 2020.

13. Chinazzi, M., Davis, J., Ajelli, M., Gioannini, C., Litvinova, M., Merler, S. y Piontti, A., and et. al, "The effect of travel restrictions on the spread of the 2019 novel coronavirus (COVID-19) outbreak," Science, merican Association for the Advancement of Science, 2020.

14. Atkeson, A., "What will be the economic impact of COVID-19 in the US? Rough estimates of disease scenarios," National Bureau of Economic Research, 2020.

15. Peng, L., Yang, W., Zhang, D. Zhuge, C., and Hong, L., "Epidemic analysis of COVID-19 in China by dynamical modeling,"arXiv preprint arXiv:2002.06563, 2020.

16. Rocklv, J. and Sjodin, H., and Wilder-Smith, A., "COVID-19 outbreak on the Diamond Princess cruise ship: estimating the epidemic potential and effectiveness of public health countermeasures," Journal of travel medicine, Oxford University Press, 2020.

17. , H., "Estimating epidemic exponential growth rate and basic reproduction number," Journal of Infectious Disease Modelling, volume 5, pages 129-141, 2020, doi = https://doi.org/10.1016/j.idm.2019.12.009.

18. Tani, U. Y., Taiti Harth, U. A., Pintor, TRF., de Cássia, PS., Marco, M., and Fernando, GJ., "Growth Rate and Acceleration Analysis of the COVID-19 Pandemic Reveals the Effect of Public Health Measures in Real Time," Frontiers in Medicine Journal, volume 7, pages 247, 2020, doi=10.3389/fmed.2020.00247.

19. Merow, C., and C. Urban, M., "Seasonality and uncertainty in global COVID-19 growth rates," Proceedings of the National Academy of Sciences Nov 2020, 117 (44) 27456-27464; doi: 10.1073/pnas.2008590117.

20. Unwin, H.J.T., Mishra, S., Bradley, V.C. et al., "State-level tracking of COVID-19 in the United States," Nat Commun 11, 6189 (2020). https://doi.org/10.1038/s41467-020-19652-6.

21. Abouk, R, and Heydari, B., "The Immediate Effect of COVID-19 Policies on Social Distancing Behavior in the United States," Public Health Reports doi: 10.1177/0033354920976575.

22. State of Illinois Coronavirus response, "COVID-19 CORONAVIRUS PANDEMIC", Online, https://coronavirus.illinois.gov/, Accessed on: February 2021.

23. Worldometer, "COVID-19 CORONAVIRUS PANDEMIC", Online, https://www.worldometers.info/coronavirus, Accessed on: February 2021.

24. The United States Department of Health and Human Service, "COVID-19 State and County Policy Orders", Online, https://healthdata.gov/dataset/covid-19-state-and-county-policy-orders, Accessed on: February 2021. 


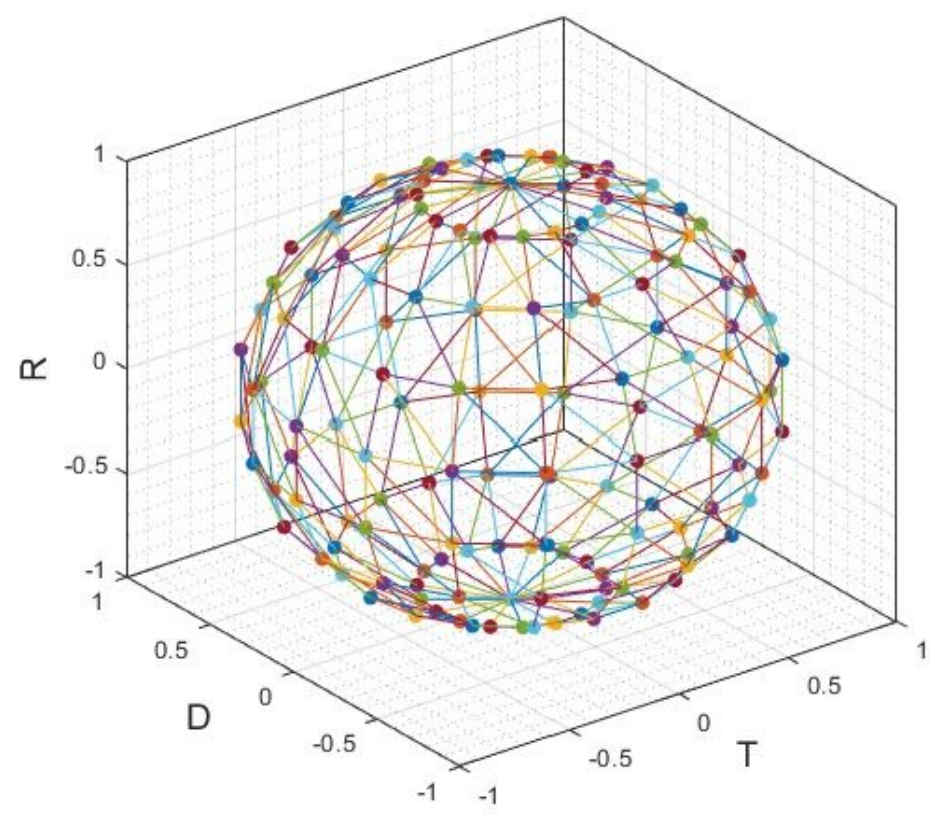

(a)

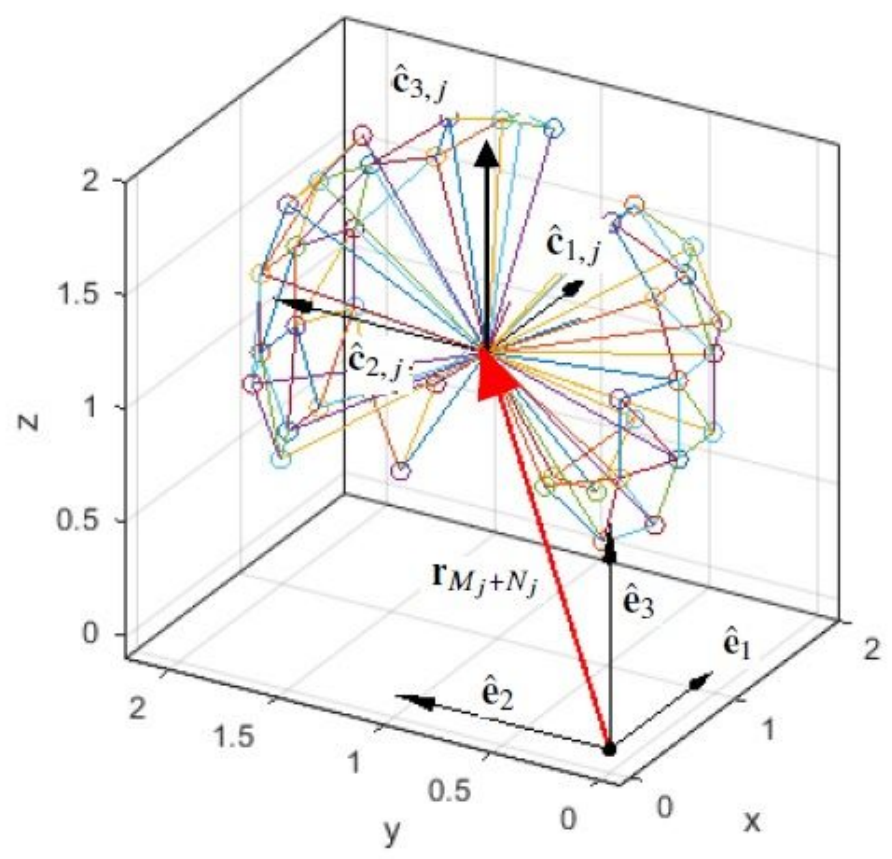

(b)

\section{Figure 1}

(see Manuscript file for figure legend)

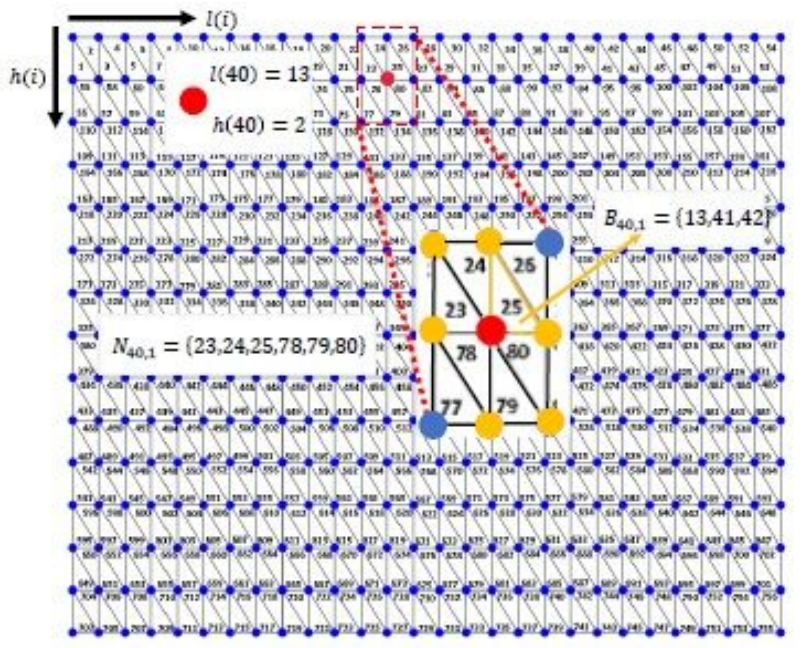

(a)

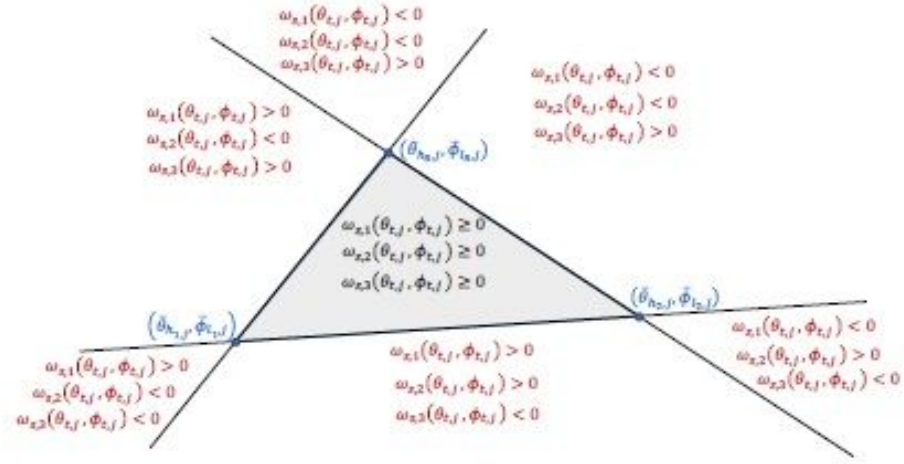

(b)

\section{Figure 2}

(see Manuscript file for figure legend) 


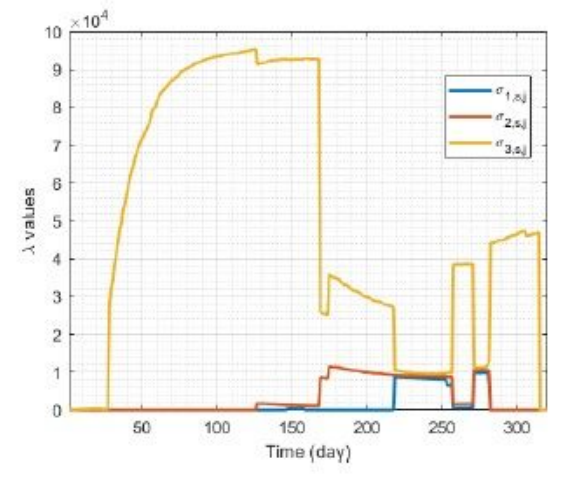

(a)

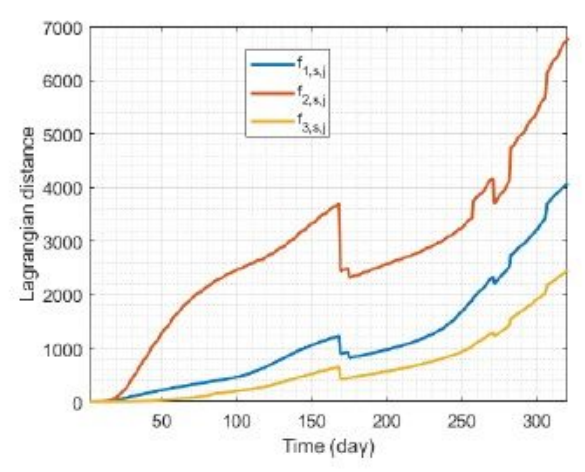

(b)

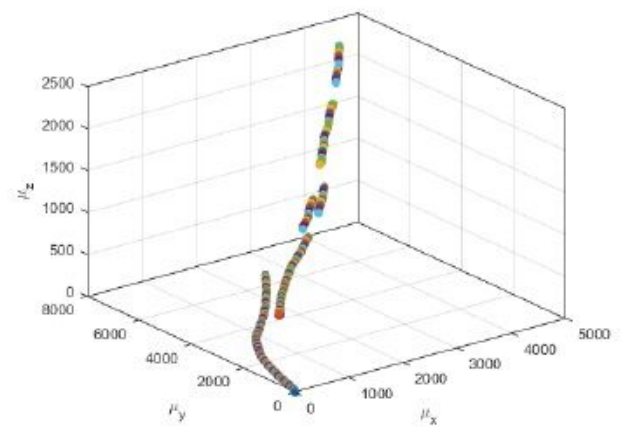

(c)

Figure 3

(see Manuscript file for figure legend)

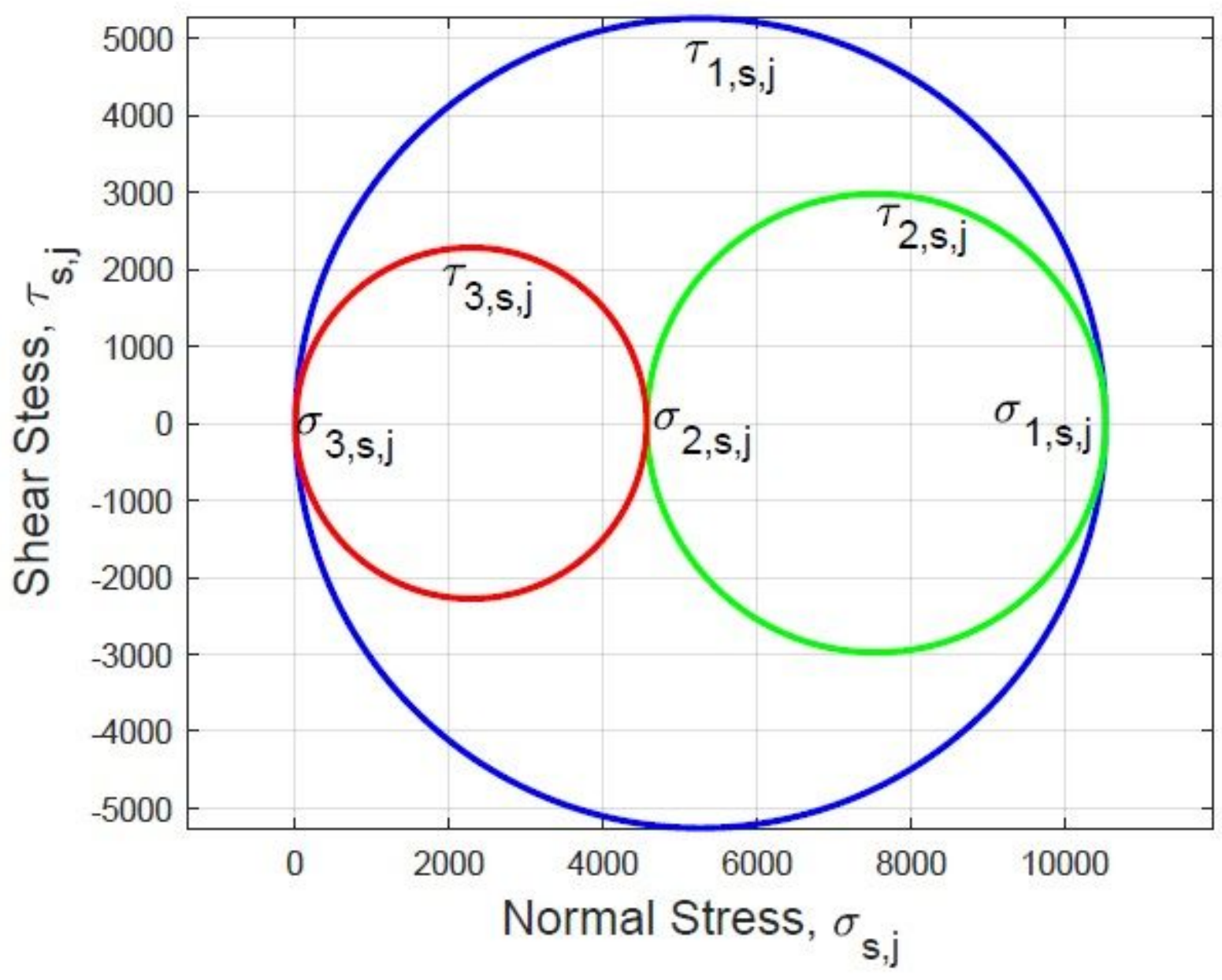

Figure 4

(see Manuscript file for figure legend) 


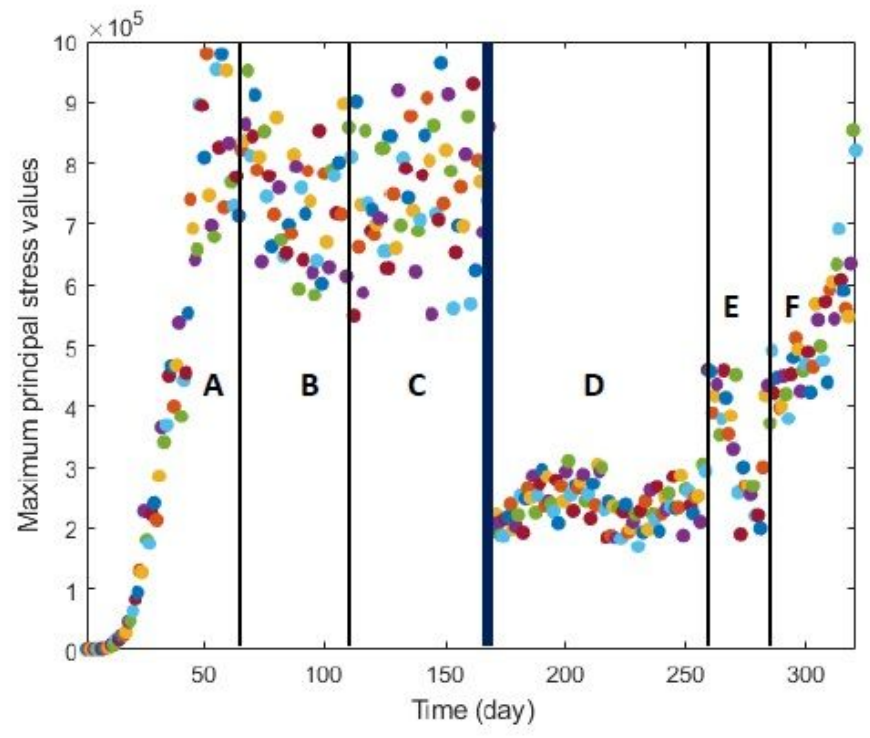

(a)

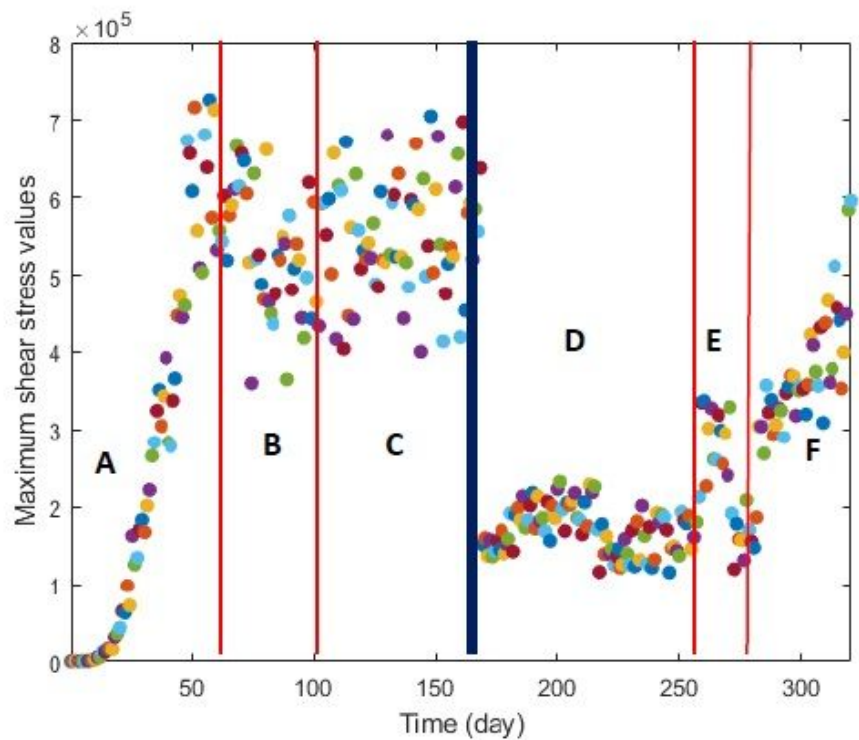

(b)

Figure 5

(see Manuscript file for figure legend)

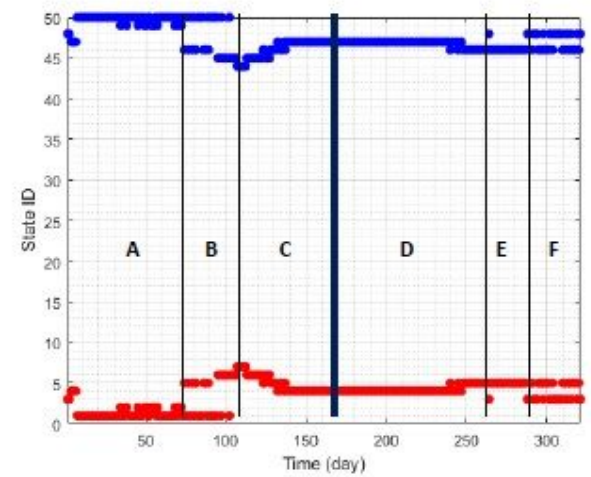

(a)

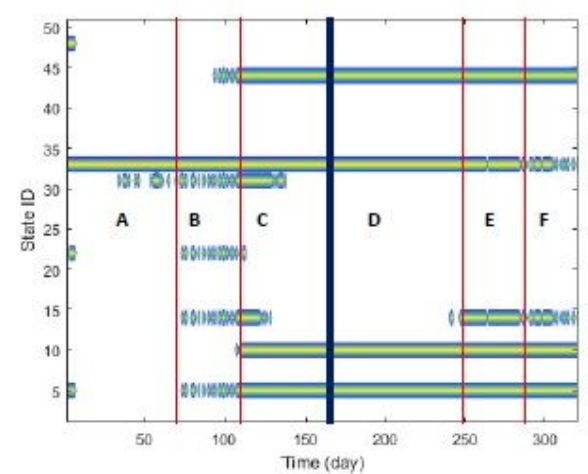

(b)

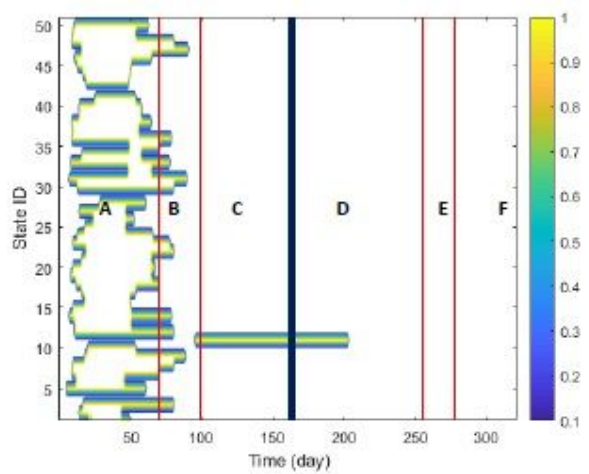

(c)

Figure 6

(see Manuscript file for figure legend) 